\title{
RESEARCH
}

Open Access

\section{Plasma $A \beta 42 / 40$ ratio alone or combined with FDG-PET can accurately predict amyloid-PET positivity: a cross-sectional analysis from the AB255 Study}

Virginia Pérez-Grijalba', Javier Arbizu², Judith Romero', Elena Prieto², Pedro Pesini ${ }^{1 *}$ (D, Leticia Sarasa1', Fernando Guillen², Inmaculada Monleón', Itziar San-José', Pablo Martínez-Lage³, Josep Munuera4, Isabel Hernández ${ }^{5,6}$, Mar Buendía ${ }^{5}$, Oscar Sotolongo-Grau ${ }^{5}$, Montserrat Alegret ${ }^{5,6}$, Agustín Ruiz ${ }^{5,6}$, Lluis Tárraga ${ }^{5,6}$, Mercè Boada ${ }^{5,6}$, Manuel Sarasa ${ }^{1}$ and The AB255 Study Group ${ }^{7}$

\begin{abstract}
Background: To facilitate population screening and clinical trials of disease-modifying therapies for Alzheimer's disease, supportive biomarker information is necessary. This study was aimed to investigate the association of plasma amyloid-beta (AB) levels with the presence of pathological accumulation of $A \beta$ in the brain measured by amyloid-PET. Both plasma Aß42/40 ratio alone or combined with an FDG-PET-based biomarker of neurodegeneration were assessed as potential AD biomarkers.
\end{abstract}

Methods: We included 39 cognitively normal subjects and 20 patients with mild cognitive impairment from the AB255 Study who had undergone PiB-PET scans. Total A 40 and A 42 levels in plasma (TP42/40) were quantified using ABtest kits. Subjects were dichotomized as A $A \beta-P E T$ positivity was assessed by logistic regression and receiver operating characteristic analyses. Combination of plasma A $\beta$ biomarkers and FDG-PET was further assessed as an improvement for brain amyloidosis detection and diagnosis classification.

Results: Eighteen (30.5\%) subjects were AB-PET positive. TP42/40 ratio alone identified A $\beta$-PET status with an area under the curve $(A \cup C)$ of 0.881 (95\% confidence interval $[\mathrm{Cl}]=0.779-0.982$ ). Discriminating performance of TP42/40 to detect AB-PET-positive subjects yielded sensitivity and specificity values at Youden's cutoff of $77.8 \%$ and $87.5 \%$, respectively, with a positive predictive value of 0.732 and negative predictive value of 0.900 . All these parameters improved after adjusting the model for significant covariates. Applying TP42/40 as the first screening tool in a sequential diagnostic work-up would reduce the number of A $\beta$-PET scans by $64 \%$. Combination of both FDG-PET scores and plasma A $\beta$ biomarkers was found to be the most accurate A $\beta$-PET predictor, with an AUC of 0.965 ( $95 \%$ $\mathrm{Cl}=0.913-0.100)$.

Conclusions: Plasma TP42/40 ratio showed a relevant and significant potential as a screening tool to identify brain $A \beta$ positivity in preclinical and prodromal stages of Alzheimer's disease.

Keywords: Plasma, Amyloid-beta, FDG-PET, Biomarker, Preclinical Alzheimer's disease, Amyloid-PET, Mild cognitive impairment

* Correspondence: ppesini@araclon.com

${ }^{1}$ Araclon Biotech S.L., Vía Hispanidad 21, 50009 Zaragoza, Spain

Full list of author information is available at the end of the article

(c) The Author(s). 2019 Open Access This article is distributed under the terms of the Creative Commons Attribution 4.0 International License (http://creativecommons.org/licenses/by/4.0/), which permits unrestricted use, distribution, and reproduction in any medium, provided you give appropriate credit to the original author(s) and the source, provide a link to the Creative Commons license, and indicate if changes were made. The Creative Commons Public Domain Dedication waiver (http://creativecommons.org/publicdomain/zero/1.0/) applies to the data made available in this article, unless otherwise stated. 


\section{Background}

Alzheimer's disease (AD) is a progressive neurodegenerative disorder and the most common cause of dementia. Accumulating data from clinical research support that the $\mathrm{AD}$ pathophysiologic process starts decades before the onset of clinical symptoms [1-3]. The disease develops in a continuum from a preclinical stage in which amyloid pathology has been defined as the earliest Alzheimer's pathological changes [3-5]. Thus, individuals at the preclinical or prodromal AD stages represent an important target group in the context of clinical trials and population screening. New diagnostic procedures can identify measurable brain changes by positron emission tomography (PET) and cerebrospinal fluid (CSF) analysis, even at the preclinical stage. These procedures have been incorporated in the National Institute of Aging-Alzheimer's Association A/T/(N) research framework for the biological definition of AD [5].

A large number of clinical studies very consistently show that these neuroimaging or CSF biomarkers provide relevant information for the diagnosis. Yet, bloodbased biomarkers are the desirable tool for large-scale assessments of patients either in clinical research or, eventually, in primary clinical settings, due to their costeffectiveness and easiness of procedures [6].

Despite previous studies yielding contradictory results [7-9], recent works have shown an association of low plasma A $\beta 42 / 40$ ratio with AD [10-13]. Plasma A $\beta 42 / 40$ ratio has also demonstrated value in detecting brain $A \beta$ pathological changes [14-16], even using different methodological approaches as mass spectrometry (MS) and new-generation immunological methods [17-19]. Our group has been largely working on the development of reliable and informative plasma biomarkers based on $A \beta$ measurements [20]. We have previously reported a consistent association between a low TP42/40 ratio and both clinical MCI diagnosis [21] and $\mathrm{A} \beta$ accumulation in the brain $[22,23]$. Reduced TP42/40 levels were also found to predict higher rates of $A \beta$ accumulation in the brain [22, 24]. Furthermore, it has been recently reported that lower TP42/40 ratio levels were also associated with increased cortical uptake of the [18F]Flortaucipir tau-PET marker in AD-related regions [25].

PET imaging, using the most widely available radiotracer ([18F]fluorodeoxyglucose PET, FDG-PET) as a measure of cerebral glucose metabolism, is a marker of neurodegeneration that has been established as a sensitive tool for detecting neuronal dysfunction [26]. FDGPET diagnostic performance could be upgraded by the use of quantitative indices that have been developed to account for inter-observer variability and to support challenging evaluation [27-29]. Arbizu et al. have previously described FDG-based automated quantitative scores, such as the AD conversion score (AD-Conv score), which has proved to predict and detect $\mathrm{AD}$ dementia with good diagnostic performance [30].

This work was primarily aimed to investigate the potential value of the TP42/40 ratio as a screening tool for brain A $\beta$-PET positivity in a population of $59 \mathrm{CN}$ and amnesic MCI (a-MCI) patients. This population was partially explored in a previous work in which clinical performance of TP42/40 was evaluated (considering clinical diagnosis as the gold standard), together with a general assessment of the correlation of TP42/40 with CSF and A $\beta$-PET biomarkers. With this study, we pursued confirming previous results from our own group in an independent AIBL population [22], focusing in the discriminating performance of TP42/40 in detecting $\mathrm{A} \beta$ positivity in the brain. Secondarily, we have explored the potential value added by the combination of $A \beta$ plasma levels and $\mathrm{AD}$-Conv score to improve prediction of $\mathrm{A} \beta$ PET status at early disease stages. Furthermore, in the framework of the new biological concept of AD based in the $\mathrm{A} / \mathrm{T} /(\mathrm{N})$ system [5], we also explored if combination of both the TP42/40 plasma ratio, as a $\beta$-amyloid marker (A), and $\mathrm{AD}$-Conv score, reflecting neurodegeneration $(\mathrm{N})$, might help in clinical assessment at early stages.

\section{Methods \\ Participants}

The AB255 Study is a multicenter longitudinal study with evaluations of the cognitive status of individuals at 0,12 , and 24 months, including 83 cognitively normal (CN) and 145 age-paired subjects with probable a-MCI [31], all over 64 years of age. The study was designed to evaluate the potential of blood-based $A \beta$ biomarkers to detect AD. A complete description of the AB255 Study protocols and population details have been previously referred [21]. Clinical diagnosis of each participant was performed using an extensive neurological examination [32] and a battery of neuropsychological tests, including evaluation of global cognition using the Mini-Mental State Examination [33, 34] and verbal learning and memory by The Word List Learning test from the Wechsler Memory Scale-Third Edition (WMS-III) [35], delayed recall and a recognition task without list of interference [36], and the Free and Cued Selective Reminding Test (FCSRT) [37]. A subpopulation of 39 cognitively normal subjects and 20 patients with a-MCI who had undergone PiB-PET scans and fulfilled criteria for inclusion were considered for the present work. Participants were all recruited and assessed from 2010 to 2013 at The Memory Clinic from Fundació ACE (Barcelona, Spain) [38].

\section{Plasma analyses}

EDTA blood was obtained after overnight fasting, and samples were immediately cooled to $2-8{ }^{\circ} \mathrm{C}$ until processed within $30 \mathrm{~h}$ of collection by centrifugation at 
$2500 \times g$ for $15 \mathrm{~min}$ at $4{ }^{\circ} \mathrm{C}$. Plasma was appropriately aliquoted in polypropylene tubes and stored at $-80^{\circ} \mathrm{C}$ until analysis, avoiding freeze-thaw cycles. ABtest 40 and ABtest42 ELISA kits (Araclon Biotech Ltd., Spain) were used for the quantification of total $A \beta 40$ and $A \beta 42$ in plasma after proprietary treatment of the sample. The specific analytical procedures and performance characteristics of these tests are described elsewhere [20]. Samples were randomized and encoded by an external CRO to guarantee the validity of results.

\section{PiB-PET analysis}

Cortical A $\beta$ burden was assessed by PET using $11 \mathrm{C}$ Pittsburg compound B (PiB-PET). Detailed procedures of neuroimaging acquisition and analysis are described by Espinosa et al. [31]. Imaging data were analyzed using the Fundació ACE Pipeline for Neuroimaging Analysis, available at http://detritus.fundacioace.com/. Participants were classified as $\beta$-amyloid positive $(\mathrm{PET}-\mathrm{A} \beta(+))$ or $\beta$ amyloid negative (PET-A $\beta(-))$ with relation to a cutoff of 1.4 SUVR in PiB-PET scans [39].

\section{FDG-PET analysis}

PET acquisition was performed after $4 \mathrm{~h}$ of fasting and once it was confirmed that blood glucose levels were below $110 \mathrm{mg} / \mathrm{dl}$. The dose of ${ }^{18} \mathrm{FDG}$ was established according to weight $(150 \mu \mathrm{Ci} / \mathrm{kg})$, being the standard adult dose $10 \mathrm{mCi}(370 \mathrm{MBq})$ in a volume of $1-10 \mathrm{ml}$ saline. Dosimetry was established in accordance with ICRP 53. For adults, critical organ dose (bladder) was $0.16 \mathrm{mGy} /$ $\mathrm{MBq}$ and effective dose was $0.019 \mathrm{mSv} / \mathrm{MBq}$. Acquisition started $40 \mathrm{~min}$ after ${ }^{18} \mathrm{FDG}$ administration and lasted for $20 \mathrm{~min}$. FDG-PET scans were analyzed following the procedure previously described by Arbizu et al. [30]. Using this automated voxel-based analytical method, quantitative indices were developed to compute the hypometabolic pattern of each subject. The AD-Conv score integrates the information provided by PET into a multivariate model including age, gender, Mini-Mental State Examination (MMSE) score, and APOE $\varepsilon 4$ genotype.

\section{APOE genotyping}

Genomic DNA was isolated from EDTA blood. Target DNA was amplified by PCR and digested with the restriction enzyme HhaI. APOE genotyping was carried out by subsequent restriction analysis of the pattern of fragments obtained after electrophoresis in a polyacrylamide gel [40].

\section{Statistical analysis}

Statistical analysis was performed using SPSS version 22 for Windows (SPSS Inc., Chicago, IL). A probability level of $p<0.05$ was considered statistically significant. Total in plasma $\mathrm{A} \beta 42 / 40$ ratio (TP42/40) and $\mathrm{AD}-\mathrm{Conv}$ score were used as the predictive variables. Demographic characteristics and biomarker levels were first compared between groups using chi-squared and Mann-Whitney tests as appropriate. Statistically significant differences in TP42/40 levels between A $\beta$-PET(+) and A $\beta$-PET(-) individuals were evaluated using generalized linear regression models (GLM) adjusted for significant demographic covariates (age, APOE genotype, and clinical group). Correlation of quantitative measures of both plasma TP42/40 ratio and AD-Conv score with A $\beta$-PET SUVR was assessed by Spearman rank correlation analyses.

The association of TP42/40 and AD-Conv score with PET-based abnormal amyloid status was further investigated using logistic regression followed by receiver operating characteristic (ROC) curve analyses. Predicted values of binary logistic regression models were used to combine variables in ROC analysis. All TP42/40 models were adjusted for age, APOE genotype (APOE\&4 allele carriers versus non-carriers), and clinical diagnosis. ADConv score was determined by a multivariate model already including age and APOE $\varepsilon 4$ genotype, so it was not readjusted for these covariates in the analysis. Plasma and FDG-PET biomarkers were evaluated alone or in combination, so that improvement in classification performance using both amyloid and neurodegeneration information derived from them was assessed. Youden's index maximizing cutoffs were considered for evaluation of biomarker performance in detecting brain $A \beta$ positivity, either individually or combined. This same procedure was applied to the total AB255 population to assess TP42/40 and AD-Conv score clinical discrimination ability ( $\mathrm{CN}$ versus a-MCI). For visualization purposes, heat maps representing predicted probability of brain $A \beta$ positivity depending on the chosen TP42/40 cutoff were depicted with regard to age and APOE genotype.

\section{Results}

Demographic characteristics of the population included in this study are summarized in Table 1. Eighteen (30.5\%) individuals were classified as A $\beta$-PET(+), and most of them were clinically diagnosed as a-MCI (14 a$\mathrm{MCI} \mathrm{A} \beta-\mathrm{PET}(+)$ versus 6 a-MCI A $\beta$-PET(-)). Likewise, 35 out of the $39 \mathrm{CN}$ individuals were classified as $\mathrm{A} \beta$ $\operatorname{PET}(-)$. Comparing $\mathrm{A} \beta-\mathrm{PET}(+)$ and $\mathrm{A} \beta-\mathrm{PET}(-)$ groups, subjects with abnormal A $\beta$-PET status were on average older and were more frequently APOE 4 carriers. Age, APOE 4, and clinical diagnosis were significantly different between groups, whereas no statistically significant differences were found with regard to gender or education level (Table 1). Both AD-Conv score and TP42/40 were significantly different between A $\beta$-PET groups (Mann-Whitney $p<0.001$ ) and correlated with A $\beta$-PET SUVR measures (TP42/40 $r_{\mathrm{s}}=-0.464 ;$ ADConv score $r_{\mathrm{s}}=0.581$; both $\left.p<0.001\right)$. Figure 1 shows 
Table 1 Demographic and biomarker features of this population

\begin{tabular}{|c|c|c|c|}
\hline & A $\beta$-PET (-) & Aß-PET (+) & $p$ value \\
\hline$N(\%)$ & $41(69.5 \%)$ & $18(30.5 \%)$ & \\
\hline Age, mean (SD) & $71.6(4.11)$ & $75.2(5.65)$ & p 0.014 \\
\hline Gender Female, $n(\%)$ & $18(43.9 \%)$ & $9(50.0 \%)$ & p 0.665 \\
\hline APOE4 carriers, $n(\%)$ & $6(14.6 \%)$ & $12(66.7 \%)$ & $p<0.001$ \\
\hline Education level, mean years (SD) & $12.33(3.96)$ & $10.56(4.44)$ & p 0.113 \\
\hline $\mathrm{CN} / \mathrm{a}-\mathrm{MCl}, n$ (\% a-MCl) & $35 / 6(14.6 \%)$ & 4/14 (77.8\%) & $p<0.001$ \\
\hline TP42/40, mean (SD) & $0.1329(0.0208)$ & $0.0997(0.0197)$ & $p<0.001$ \\
\hline AD-Conv score, mean (SD) & $0.133(0.162)$ & $0.384(0.259)$ & $p<0.001$ \\
\hline
\end{tabular}

$p$ value was obtained from Mann-Whitney or chi-squared tests as appropriate. SD standard deviation, $C N$ cognitively normal, $a-M C l$ amnestic mild cognitive impairment, $A \beta$ amyloid-beta, TP42/40 total plasma $A \beta 42 / 40$ ratio, $A D$-Conv score $A D$ conversion score based on FDG-PET

these associations including information of the clinical status of each participant.

Plasma TP42/40 ratio was in average $25 \%$ lower in PET-A $\beta(+)$ subjects compared to PET-A $\beta(-)$ individuals (Table 1 and Fig. 2). After adjusting for significant demographic covariates (age, APOE 4, and clinical diagnosis), association of TP42/40 with A $\beta$-PET status remained statistically significant (GLM $\beta=87.67, p=$ 0.005). ROC analysis revealed an AUC of 0.881 (95\% confidence interval $[\mathrm{CI}]$ 0.779-0.982) for the prediction of A $\beta$-PET positivity using TP42/40 alone (Fig. 3a). The Youden's cutoff of plasma TP42/40 ratio was 0.1049 and yielded a sensitivity of $77.8 \%$ and a specificity of $87.5 \%$ in detecting PET-A $\beta(+)$ subjects (Table 2). When studying independently $\mathrm{CN}$ and a-MCI subjects, we found excellent performance of $\mathrm{TP} 42 / 40$ in the $\mathrm{CN}$ group (AUC unadjusted TP42/40 0.957, 95\% CI 0.890-1.000), although it declined in the a-MCI group (AUC unadjusted TP42/40 0.814, 95\% CI 0.617-1.000) (Table 2).

When adjusting TP42/40 with relevant covariates (age, APOE genotype, and clinical group), discrimination was improved yielding an AUC of 0.963 (95\% CI $0.917-$ 1.000). Sensitivity, specificity, and NPV were all over 0.920 (Fig. 3b, Table 2). We used the logistic regression model to predict probabilities of A $\beta$-PET positivity with regard to age in both APOE \&4 non-carriers (Fig. 4a) and carriers (Fig. 4b). This figure represents a statistical inference since the actual study population does not cover all the age ranges represented. As expected, the predicted probability increased with age and was always higher in APOE \&4 carriers compared to non-carriers. Low TP42/40 ratio considerably augmented likelihood of abnormal A $\beta$-PET status for every genotype and age condition. Indeed, the high NPV and low LR- characterized TP42/40 as an ideal marker for pre-screening. Considering a hypothetical clinical trial targeting $A \beta$ PET-positive subjects, a recruitment procedure based in A $\beta$-PET alone would require 3.3 PET scans per successful recruitment. However, following a sequential strategy including a TP42/40 pre-screening stage would require 1.2 A $\beta$-PET scans per successful recruitment, implying a 64\% saving in PET scans.
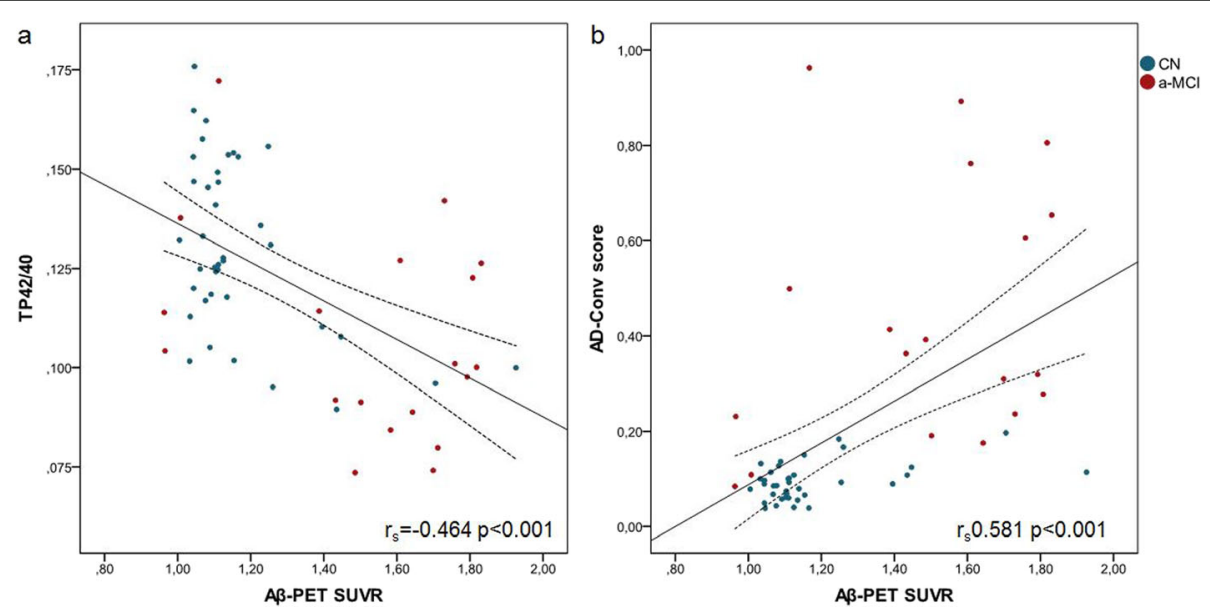

Fig. 1 Correlation of TP42/40 and AD-Conv score with AB-PET. Scatterplots of plasma TP42/40 ratio (a) and AD-Conv score (b) levels with regard to PiB-PET. Blue: cognitively normal (CN) subjects. Red: amnestic mild cognitive impairment (a-MCI) individuals. Both TP42/40 and AD-Conv score biomarkers showed a significant correlation with AB-PET, although in inverse directions. $r_{\mathrm{s}}$ Spearman rank correlation coefficient 


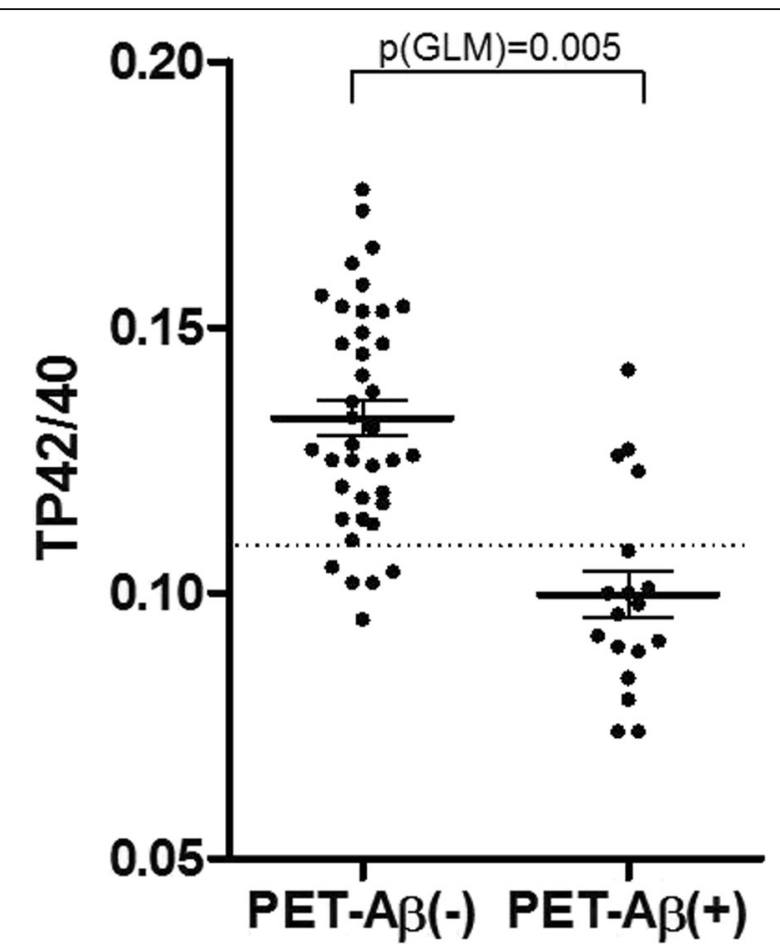

Fig. 2 Distribution of TP42/40 levels between AB-PET groups. Dots represent the individual TP42/40 ratio obtained for each patient with regard to their AB-PET status. Continuous lines represent mean TP42/40 values and standard mean error. The dashed line represents the Youden's cutoff (0.1094) PET-A $\beta$ positivity discrimination using TP42/40 alone. $p$ value from the generalized linear model (GLM) was also included

AD-Conv score was also evaluated as a predictor of A $\beta$-PET positivity in this cohort, showing good diagnostic performance (AUC 0.898, 95\% CI 0.817-0.979) (Fig. 3b), and sensitivity and specificity values at maximum Youden's cutoff of $100 \%$ and $67.5 \%$, respectively
(Table 2). Thus, we hypothesized that a combination of both plasma TP42/40 and neurodegeneration (FDGPET) biomarkers could outperform the single-predictor models in detecting A $\beta-\mathrm{PET}(+)$ subjects.

The combined model of plasma $A \beta$ and neurodegeneration biomarkers (AdjTP42/40+AD-Conv score) improved performance of both individual biomarkers and covariates alone (Fig. $3 \mathrm{c}$ ) to discriminate A $\beta$-PET status. AUC for this model was found to be 0.965 (95\% CI $0.921-1.000)$, with very high sensitivity and specificity (>92\%) and NPV of 1.00 (Table 2).

On the other hand, TP42/40 performed poorly at discriminating between $\mathrm{CN}$ and a-MCI patients in the whole AB255 study population (AUC $=0.639,95 \%$ CI 0.564-0.714). Nevertheless, combining TP42/40 with AD-Conv score improved subjects' clinical classification up to an AUC of 0.915 (95\% CI 0.875-0.955). The effect is mainly driven by AD-Con score which by itself alone presented an AUC of 0.893 (95\% CI 0.849-0.937).

\section{Discussion}

In this study, we aimed at assessing the potential value of TP42/40 ratio in the prediction of brain $A \beta$ pathology measured by $\mathrm{A} \beta-\mathrm{PET}$ in a preclinical and prodromal $\mathrm{AD}$ cohort of 59 patients from the AB255 Study. In addition, the present study evaluated the potential benefit of the combination of amyloid and neurodegeneration biomarkers, reflected by plasma TP42/40 and the FDGPET-derived AD-Conv score [30]. Both individually and combined, these biomarkers significantly detected $A \beta$ pathology in the brain. Combination of adjusted TP42/ 40 ratio and AD-Conv score was found to be the most accurate A $\beta$-PET predictor, with sensitivity of $100 \%$ and specificity of $92.3 \%$.
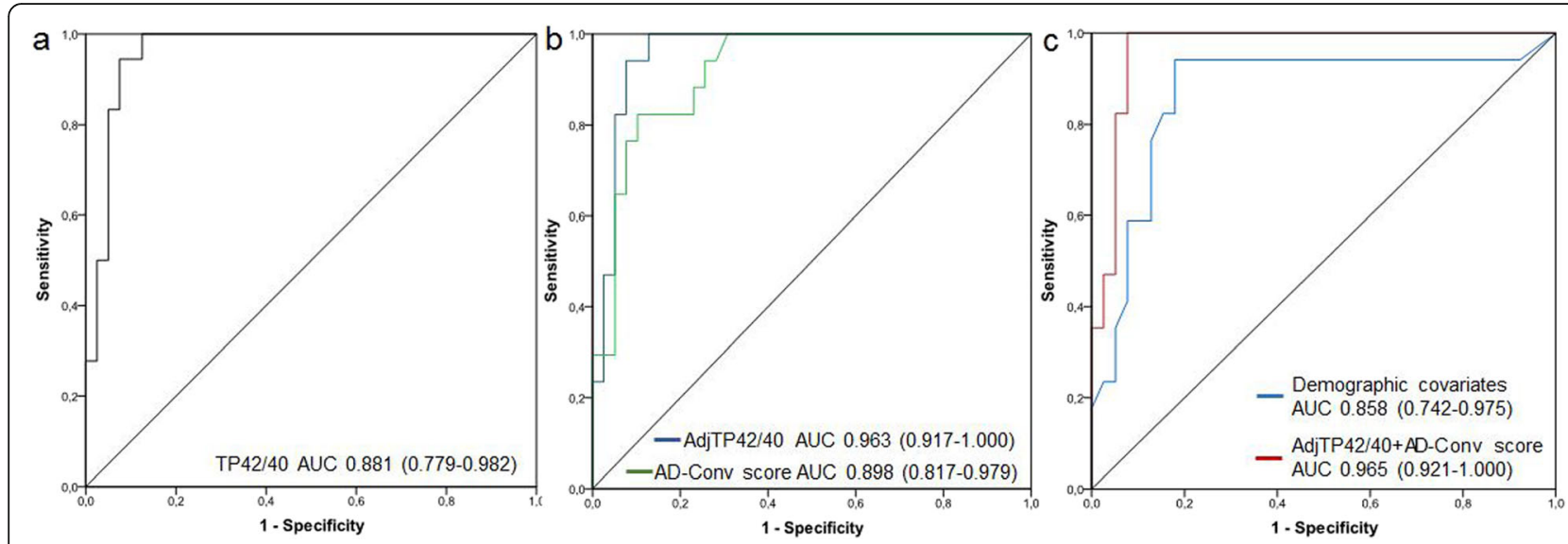

Fig. 3 Receiver operating characteristic (ROC) curves discriminating A $\beta-P E T(+)$ from A $-P E T(-)$ subjects. ROC curve and the corresponding area

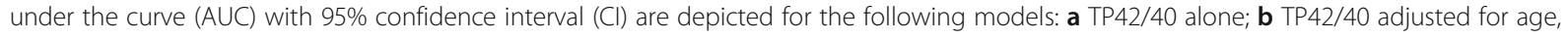
APOE genotype, and clinical group (AdjTP42/40) and AD-Conv score; c models including only significant demographic covariates (age, APOE genotype, and clinical group) and the combined model of amyloidosis and neurodegeneration (AdjTP42/40+AD-Conv score) 
Table 2 Diagnostic performance of individual and combined models in detecting Aß-PET positivity at Youden's cutoff

\begin{tabular}{|c|c|c|c|c|c|c|}
\hline & Sensitivity & Specificity & PPV & NPV & LR+ & LR- \\
\hline \multicolumn{7}{|l|}{ Whole population $(N=59)$} \\
\hline TP42/40 & 0.778 & 0.875 & 0.732 & 0.900 & 6.22 & 0.25 \\
\hline Age+APOE4+Clinical Group & 0.944 & 0.805 & 0.680 & 0.945 & 4.84 & 0.07 \\
\hline TP42/40+Age+APOE4+Clinical Group & 0.944 & 0.925 & 0.847 & 0.974 & 12.59 & 0.06 \\
\hline AD-Conv score & 1.000 & 0.675 & 0.706 & 1.000 & 3.08 & 0.00 \\
\hline AdjustedTP42/40+AD-Conv score & 1.000 & 0.923 & 0.851 & 1.000 & 13.00 & 0.00 \\
\hline \multicolumn{7}{|l|}{ Only CN $(N=39)$} \\
\hline TP42/40 & 1.000 & 0.886 & 0.500 & 1.000 & 8.75 & 0.00 \\
\hline Age+APOE4 & 0.750 & 0.943 & 0.600 & 0.971 & 12.12 & 0.26 \\
\hline TP42/40+Age+APOE4 & 1.000 & 1.000 & 1.000 & 1.000 & - & 0.00 \\
\hline AD-Conv score & 1.000 & 0.765 & 0.327 & 1.000 & 4.25 & 0.00 \\
\hline AdjustedTP42/40+AD-Conv score & 1.000 & 1.000 & 1.000 & 1.000 & - & 0.00 \\
\hline \multicolumn{7}{|l|}{ Only a-MCI $(N=20)$} \\
\hline TP42/40 & 0.714 & 1.000 & 1.000 & 0.653 & - & 0.29 \\
\hline Age+APOE4 & 0.357 & 0.833 & 0.799 & 0.411 & 2.14 & 0.77 \\
\hline TP42/40+Age+APOE4 & 0.643 & 1.000 & 1.000 & 0.601 & - & 0.36 \\
\hline AD-Conv score & 0.846 & 0.500 & 0.759 & 0.636 & 1.70 & 0.31 \\
\hline AdjustedTP42/40+AD-Conv score & 0.615 & 1.000 & 1.000 & 0.583 & - & 0.38 \\
\hline
\end{tabular}

Cases in which LR+ was not quantifiable were presented as -. Prevalence considered for each group calculation was corresponding to the present cohort: $30.5 \%$ in the whole population, $10.25 \%$ in the $C N$ group, and $65 \%$ in the a-MCI group. PET PPV-positive predictive value, NPV negative predictive value, $L R+$ positive likelihood ratio, $L R-$ negative likelihood ratio

$\mathrm{A} \beta-\mathrm{PET}(+)$ patients showed significantly lower levels of TP42/40, with an average reduction of $25 \%$ compared to the A $\beta-\operatorname{PET}(-)$ group. This significant inverse association of plasma $A \beta 42 / 40$ ratio with brain $A \beta$ deposition is in accordance with previous studies [14-19]. These findings also replicate our previous results showing the potential of TP42/40 as a surrogate biomarker of $A \beta$ PET status in an independent preclinical population [22]. TP42/40 ratio adjusted for significant covariates detected A $\beta$-PET positivity in the present cohort with
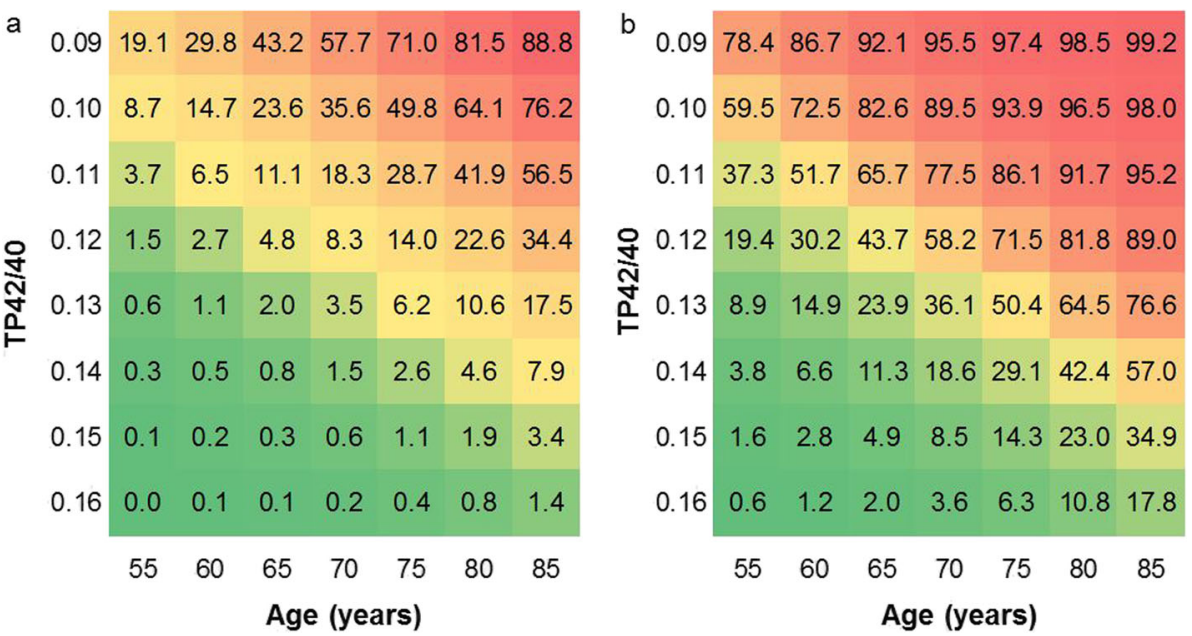

Fig. 4 Heat maps showing predicted probability of being A -PET(+) based on TP42/40 and age stratified for APOE genotype. Predicted probabilities in APOE $\mathcal{E} 4$ non-carriers (a) and APOE $\mathcal{E} 4$ carriers (b). Probabilities are displayed as percentages. Note that TP42/40 decreases upward in axis for optimal visualization purposes. It should be noted that probabilities were predicted from a logistic regression model modeling expected probabilities in the age range most likely present during pre-screening or clinical assessment. These predicted probabilities could be more accurately determined in a larger population including the complete age range 
94.4\% sensitivity and $92.5 \%$ specificity. This means that in a sequential screening scenario aiming to detect subjects with abnormal A $\beta$-PET status, a first step assessing TP42/40 would allow a $64 \%$ reduction in the number of $\mathrm{A} \beta$-PET scans. Considering the restricted availability and costs of A $\beta$-PET neuroimaging [41], this sequential strategy would imply a significant reduction of the patient burden and procedural costs, both for clinical trials and patient management at primary care settings.

Clinical performance of plasma $A \beta 42 / A \beta 40$ in the present study is comparable to that obtained using MSbased methods $[17,18]$ or new-generation immunological assays [19] and are congruent with other studies demonstrating a consistent inverse association of the plasma $A \beta 42 / A \beta 40$ with the disease $[10-13,42,43]$. Nevertheless, there still exists considerable overlap of plasma $A \beta$ ratio between diagnostic groups or amyloid status groups, which limits the use of $A \beta$ plasma ratios for clinical diagnosis at the individual level. On the other hand, clinical performance of plasma biomarkers is also hampered by the fact that $\mathrm{A} \beta$-PET classification, used as the gold standard, neither is utterly precise. In line with this, plasma TP42/40 false-positive results could correspond to a-MCI subjects who are already accumulating $A \beta$ in the brain, but have not reached the threshold for abnormal status yet. If this were the case, plasma $A \beta$ biomarkers would turn out to be abnormal before $A \beta$ PET does. Alternatively, misclassification could be due to heterogeneity of the underlying disease in a-MCI patients. When using only $\mathrm{CN}$ to explore TP42/40 potential in detecting $\mathrm{A} \beta-\mathrm{PET}+$ subjects, results are better compared to the a-MCI group or the mixed population. Adjusted TP42/40 can accurately detect the 4 AB-PET+ individuals in the cohort of $39 \mathrm{CN}$, which confirms the preclinical potential of this biomarker when detecting $\mathrm{A} \beta$ abnormality in the brain, detected also in previous work from our group [22]. However, due to the small size of the $\mathrm{CN}$ and a-MCI groups in the present population, together with the different prevalence of $A \beta$-PET positivity, these findings should be taken cautiously and further replicated.

AD-Conv score has proven a significant correlation with $\mathrm{A} \beta$-PET and a good performance in detecting brain $A \beta$ pathology within this cohort, being the AUC values obtained within the range previously defined [30]. These results reinforce the suitability of quantitative indices derived from automated FDG-PET measures replacing standard qualitative brain FDG-PET imaging [27-29].

Previous studies evaluating the association of FDGPET and A $\beta$-PET measures have shown discrepant results. Several works did not find a correlation in AD [44-46], MCI [47], and CN individuals [48, 49], although it was found in other cohorts [50-54]. Another study including both $\mathrm{CN}$ and $\mathrm{MCI}$ individuals showed a significant correlation between PiB-PET and FDG-PET [55], corresponding well to our findings.

Only few studies have explored combination of FDGPET and A $\beta$ biomarkers, mostly in CSF and strictly focused on conversion to $\mathrm{AD}$ dementia [43, 46-48]. Interestingly, combination of TP42/40 and AD-Conv score improves subjects' clinical classification into $\mathrm{CN}$ or a-MCI. As could be expected, the effect is mainly driven by the AD-Conv score which itself integrates into a multivariate model FDG-PET neurodegeneration (downstream to $\beta$ amyloid changes as per the amyloid cascade hypothesis) with APOE genotype, MMSE score, age, and gender. This approach is in agreement with the recent research framework based on the $\mathrm{A} / \mathrm{T} /(\mathrm{N})$ system [5], and our findings support the suitability of a biological definition of $\mathrm{AD}$, measured by biomarkers that are reflecting different processes underlying AD clinical manifestations.

Larger and varied populations need to be explored in order to confirm the potential utility of FDG-PET and TP42/40 combination, which may vary depending on population characteristics and disease stage. In fact, the overall improvement of the adjusted TP42/40 model by AD-Conv score, although relevant, was relatively modest in our cohort. Another weakness of our study is the absence of a PiB-PET follow-up, which would be of great utility in ascertaining the potential prognostic value of plasma biomarkers to predict the rate of brain $A \beta$ deposition and could have provided relevant evidence for elucidating the possible source of bias in the classification performance.

\section{Conclusions}

This study demonstrates a consistent inverse association of plasma TP42/40 and FDG-PET biomarkers with A $\beta$ PET status. Findings from this work replicate our previous results in different cohorts, hence confirming TP42/40 as a useful biomarker to rule out brain $\beta$-amyloidosis in preclinical and early AD stages. Plasma TP42/40 tests may be used as an initial screening tool for patient management; only those resulting TP42/40 positives would have to undergo diagnostic confirmation by CSF analysis or PET neuroimaging. Integration of neurodegeneration and blood-based $\mathrm{A} \beta$ biomarkers could contribute to early $\mathrm{AD}$ diagnosis in compliance with the $\mathrm{A} / \mathrm{T} /(\mathrm{N})$ research framework, although it requires further validation.

\footnotetext{
Abbreviations

AD: Alzheimer's disease; PET: Positron emission tomography; CSF: Cerebrospinal fluid; CN: Cognitively normal; MCl: Mild cognitive impairment; A $\beta$-PET: Amyloid-beta PET; MS: Mass spectrometry; A $\beta$ : Amyloidbeta; TP42/40: Total in plasma Aß42/40; AIBL: Australian Imaging Biomarkers and Lifestyle Study of Aging; FDG-PET: [18F]fluorodeoxyglucose PET; ADConv score: AD conversion score (FDG-PET); a-MCl: Amnestic MCl; $\mathrm{CRO}$ : Contract research organization; ROC: Receiver operating characteristic; $\mathrm{Cl}$ : Confidence interval; GLM: Generalized linear regression models; PPV: Positive predictive value; NPV: Negative predictive value; LR: Likelihood ratio
} 


\section{Acknowledgements}

The authors would like to thank the participants and collaborators of this study and the entire AB255 Study Group.

Other AB255 Study Group collaborators: Miguel Goñi7; Francesc Pujadas8;

Alberto Villarejo9; Ana Frank10; Jordi Peña-Casanova11; Manuel Fernández12; Gerard Piñol13; Rafael Blesa14; Pedro Gil15; Luis F. Pascual16; Miquel

Aguilar17; Giovanni B Frisoni18; Jorge Matias-Guiu15; Niels Andreasen9; Carmen Antúnez20.

7. Hospital Divino Vallés, Burgos, Spain

8. Hospital Universitari Vall d'Hebrón, Barcelona, Spain

9. Hospital Doce de Octubre, Madrid, Spain

10. Hospital La Paz, Madrid, Spain

11. Hospital del Mar, Barcelona, Spain

12. CAE Oroitu Algorta, Vizcaya, Spain

13. Hospital Santa María de Lleida, Lleida, Spain

14. Hospital de la Santa Creu i Sant Pau, Barcelona, Spain

15. Hospital Clínico San Carlos, Madrid, Spain

16. Hospital Lozano Blesa, Zaragoza, Spain

17. Hospital Universitari Mútua

Terrassa, Terrassa, Spain

18. IRCCS Centro San Giovanni di Dio FBF, Brescia, Italy

19.Karolinska Institutet, Stockholm, Sweden

20. Hospital Virgen de la Arrixaca, Fundación Alzheimur, Murcia, Spain

\section{Authors' contributions}

VPG and PP carried out the data analysis and interpretation and prepared and wrote the manuscript. JR performed $A \beta$ quantification. LS contributed to the APOE analysis. IM and ISJ contributed to the study design, sample collection, and processing. JA contributed to the study design, recruitment, and FDG-PET acquisition and data analysis. IH, MA, and MB participated in the data collection and analysis. PML, JM, LT, AR, MB, and MS conceived and designed the study. All authors reviewed the manuscript. All authors read and approved the final manuscript.

\section{Funding}

This work was funded by Araclon Biotech, Fundació ACE Memory Clinic, as well as the Spanish Ministry of Health through Instituto de Salud Carlos III (Madrid) (FISS PI10/00954) and by Agència d'Avaluació de Tecnologia i Recerca Mèdiques, Departament de Salut de la Generalitat de Catalunya (RECERCALIA grant 390/06/ 2009). Araclon Biotech was the main funder of the study, participating in conceptualization, design, data collection, and manuscript preparation.

\section{Availability of data and materials}

The datasets used and/or analyzed during the current study are available from the corresponding author on reasonable request.

\section{Ethics approval and consent to participate}

The study was approved by the CEIC ethic committee 2009/5455, and all participants provided written informed consent prior to inclusion. Patient recruitment and collection protocols were in accordance with ethical standards according to WMA Declaration of Helsinki-Ethical Principles for Medical Research Involving Human Subjects.

\section{Consent for publication}

Consent for publication was provided by every participant of the study.

\section{Competing interests}

VPG, PP, JR, ISJ, LS, IM, and MS were employees of Araclon Biotech at the moment the study and data analysis were carried out. All other co-authors declare that they have no competing interests.

\section{Author details}

${ }^{1}$ Araclon Biotech S.L., Vía Hispanidad 21, 50009 Zaragoza, Spain. ${ }^{2}$ Servicio de Medicina Nuclear, Clínica Universidad de Navarra, Pamplona, Spain. ${ }^{3}$ Center for Research and Advanced Therapies and Memory Clinic, Fundación CITA-Alzheimer, San Sebastián, Spain. ${ }^{4}$ Institut de recerca Sant Joan de Déu, Hospital Infantil Sant Joan de Déu, Barcelona, Spain. ${ }^{5}$ Research Center and Memory Clinic, Fundació ACE, Institut Català de Neurociències Aplicades, Universitat Internacional de Catalunya-Barcelona, Barcelona, Spain. ${ }^{6}$ Networking Research Center on Neurodegenerative Diseases (CIBERNED),
Instituto de Salud Carlos III, Madrid, Spain. ${ }^{7}$ https://www.araclon.com/ alzheimer/abtest/current-situation/.

Received: 11 July 2019 Accepted: 22 October 2019

\section{1. -}

\section{References}

1. Buchhave P, Minthon L, Zetterberg H. Cerebrospinal fluid levels of $\beta$ amyloid $1-42$, but not of tau, are fully changed already 5 to 10 years before the onset of Alzheimer dementia. Arch Gen Psychiatry. 2012;69(1):9.

2. Jack CR, Holtzman DM. Biomarker modeling of Alzheimer's disease. Neuron. 2013;80(6):1347-58.

3. Dubois B, Hampel H, Feldman HH, Scheltens P, Aisen P, Andrieu S, et al. Preclinical Alzheimer's disease: definition, natural history, and diagnostic criteria. Alzheimers Dement. 2016;12(3):292-323.

4. Sperling RA, Aisen PS, Beckett LA, Bennett DA, Craft S, Fagan AM, et al. Toward defining the preclinical stages of Alzheimer's disease: recommendations from the National Institute on Aging-Alzheimer's Association workgroups on diagnostic guidelines for Alzheimer's disease. Alzheimers Dement. 2011;7(3):280-92.

5. Jack $C R$, Bennett DA, Blennow $K$, Carrillo MC, Dunn B, Haeberlein SB, et al. NIA-AA research framework: toward a biological definition of Alzheimer's disease. Alzheimers Dement. 2018;14(4):535-62.

6. Bateman RJ, Blennow K, Doody R, Hendrix S, Lovestone S, Salloway S. Plasma biomarkers of $\mathrm{AD}$ emerging as essential tools for drug development: an EU/US CTAD task force report. J Prev Alzheimer's Dis JPAD. 2019;6(3): 169-73.

7. Hansson $\mathrm{O}$, Zetterberg $\mathrm{H}$, Vanmechelen $\mathrm{E}$, Vanderstichele $\mathrm{H}$, Andreasson $\mathrm{U}$, Londos $E$, et al. Evaluation of plasma $A \beta 40$ and $A \beta 42$ as predictors of conversion to Alzheimer's disease in patients with mild cognitive impairment. Neurobiol Aging. 2010;31(3):357-67.

8. Lopez OL, Kuller LH, Mehta PD, Becker JT, Gach HM, Sweet RA, et al. Plasma amyloid levels and the risk of $A D$ in normal subjects in the Cardiovascular Health Study. Neurology. 2008;70(19):1664-71.

9. Lövheim $\mathrm{H}$, Elgh $\mathrm{F}$, Johansson A, Zetterberg $\mathrm{H}$, Blennow $\mathrm{K}$, Hallmans $\mathrm{G}$, et al. Plasma concentrations of free amyloid $\beta$ cannot predict the development of Alzheimer's disease. Alzheimers Dement. 2017;13(7):778-82.

10. Graff-Radford NR, Crook JE, Lucas J, Boeve BF, Knopman DS, Ivnik RJ, et al. Association of low plasma $A \beta 42 / A \beta 40$ ratios with increased imminent risk for mild cognitive impairment and Alzheimer disease. Arch Neurol. 2007;64:9.

11. Lambert J-C, Schraen-Maschke S, Richard F, Fievet N, Rouaud O, Berr C, et al. Association of plasma amyloid with risk of dementia: the prospective ThreeCity Study. Neurology. 2009;73(11):847-53.

12. Chouraki V, Beiser A, Younkin L, Preis SR, Weinstein G, Hansson O, et al. Plasma amyloid- $\beta$ and risk of Alzheimer's disease in the Framingham Heart Study. Alzheimers Dement. 2015;11(3):249-257.e1.

13. van Oijen M, Hofman A, Soares HD, Koudstaal PJ, Breteler MM. Plasma AB140 and $A \beta 1-42$ and the risk of dementia: a prospective case-cohort study. Lancet Neurol. 2006;5(8):655-60.

14. Lui JK, Laws SM, Li Q-X, Villemagne VL, Ames D, Brown B, et al. Plasma amyloid-beta as a biomarker in Alzheimer's disease: the AIBL study of aging. J Alzheimers Dis. 2010;20(4):1233-42.

15. Devanand DP, Schupf N, Stern Y, Parsey R, Pelton GH, Mehta P, et al. Plasma $A$ and PET PiB binding are inversely related in mild cognitive impairment. Neurology. 2011;77(2):125-31.

16. Rembach A, Faux NG, Watt AD, Pertile KK, Rumble RL, Trounson BO, et al. Changes in plasma amyloid beta in a longitudinal study of aging and Alzheimer's disease. Alzheimers Dement. 2014;10(1):53-61.

17. Nakamura A, Kaneko N, Villemagne VL, Kato T, Doecke J, Doré V, et al. High performance plasma amyloid- $\beta$ biomarkers for Alzheimer's disease. Nature. 2018:554(7691):249-54.

18. Ovod V, Ramsey KN, Mawuenyega KG, Bollinger JG, Hicks T, Schneider T, et al. Amyloid $\beta$ concentrations and stable isotope labeling kinetics of human plasma specific to central nervous system amyloidosis. Alzheimers Dement. 2017;13(8):841-9.

19. Janelidze S, Stomrud E, Palmqvist S, Zetterberg $H$, van Westen $D$, Jeromin A, et al. Plasma $\beta$-amyloid in Alzheimer's disease and vascular disease. Sci Rep. 2016;6(1):26801.

20. Pérez-Grijalba V, Fandos N, Canudas J, Insua D, Casabona D, Lacosta AM, et al. Validation of immunoassay-based tools for the comprehensive 
quantification of $A \beta 40$ and $A \beta 42$ peptides in plasma. J Alzheimers Dis. 2016; 54(2):751-62.

21. Pérez-Grijalba V, Romero J, Pesini P, Sarasa L, Monleón I, San-José I, et al. Plasma AB42/40 ratio detects early stages of Alzheimer's disease and correlates with CSF and neuroimaging biomarkers in the AB255 study. J Prev Alzheimers Dis. 2019;6(1):34-41.

22. Fandos N, Pérez-Grijalba V, Pesini P, Olmos S, Bossa M, Villemagne $V L$, et al. Plasma amyloid $\beta$ 42/40 ratios as biomarkers for amyloid $\beta$ cerebral deposition in cognitively normal individuals. Alzheimers Dement Diagn Assess Dis Monit. 2017:8:179-87.

23. de Rojas I, Romero J, Rodríguez-Gomez O, Pesini P, Sanabria A, PérezCordon A, et al. Correlations between plasma and PET beta-amyloid levels in individuals with subjective cognitive decline: the Fundació ACE Healthy Brain Initiative (FACEHBI). Alzheimers Res Ther. 2018;10(1):119.

24. Doecke J, Pérez-Grijalba V, Fandos N, Fowler C, Villemagne VL, Masters CL, et al. Total A $442 / A \beta 40$ ratio in plasma predict amyloid-PET status, independent of clinical AD diagnosis. Neurology. 2019; in press.

25. Risacher SL, Fandos N, Romero J, Sherriff I, Pesini P, Saykin AJ, et al. Plasma $A \beta$ levels are associated with cerebral amyloid and tau deposition. Alzheimers Dementia. 2019;11:510-9.

26. Jagust W, Reed B, Mungas D, Ellis W, Decarli C. What does fluorodeoxyglucose PET imaging add to a clinical diagnosis of dementia? Neurology. 2007;69(9):871-7.

27. Shaffer JL, Petrella JR, Sheldon FC, Choudhury KR, Calhoun VD, Coleman RE, et al. Predicting cognitive decline in subjects at risk for Alzheimer disease by using combined cerebrospinal fluid, MR imaging, and PET biomarkers. Radiology. 2013;266(2):583-91.

28. Landau SM, Harvey D, Madison CM, Koeppe RA, Reiman EM, Foster NL, et al. Associations between cognitive, functional, and FDG-PET measures of decline in AD and MCl. Neurobiol Aging. 2011;32(7):1207-18.

29. Chen K, Ayutyanont N, Langbaum JBS, Fleisher AS, Reschke C, Lee W, et al. Characterizing Alzheimer's disease using a hypometabolic convergence index. Neurolmage. 2011;56(1):52-60.

30. Arbizu J, Prieto E, Martínez-Lage P, Martí-Climent JM, García-Granero M, Lamet I, et al. Automated analysis of FDG PET as a tool for single-subject probabilistic prediction and detection of Alzheimer's disease dementia. Eur J Nucl Med Mol Imaging. 2013;40(9):1394-405.

31. Espinosa A, Alegret M, Pesini $P$, Valero S, Lafuente A, Buendía M, et al. Cognitive composites domain scores related to neuroimaging biomarkers within probable-amnestic mild cognitive impairment-storage subtype. J Alzheimers Dis. 2017;57(2):447-59.

32. Alegret $M$, Espinosa A, Valero S, Vinyes-Junqué G, Ruiz A, Hernández I, et al. Cut-off scores of a brief neuropsychological battery (NBACE) for Spanish individual adults older than 44 years old. PLoS One. 2013;8(10):e76436.

33. Folstein MF, Folstein SE, McHugh PR. "Mini-mental state". A practical method for grading the cognitive state of patients for the clinician. J Psychiatr Res. 1975;12(3):189-98.

34. Blesa R, Pujol M, Aguilar M, Santacruz P, Bertran-Serra I, Hernández G, et al. Clinical validity of the "mini-mental state" for Spanish speaking communities. Neuropsychologia. 2001;39(11):1150-7.

35. Wechsler D. WAIS-III. Wechsler adult intelligence scale-third edition. Technical manual. San Antonio: The Psychological Corporation; 1997.

36. Alegret M, Espinosa A, Vinyes-Junqué G, Valero S, Hernández I, Tárraga L, et al. Normative data of a brief neuropsychological battery for Spanish individuals older than 49. J Clin Exp Neuropsychol. 2012;34(2):209-19.

37. Buschke H. Cued recall in amnesia. J Clin Neuropsychol. 1984;6(4):433-40.

38. Boada M, Tárraga L, Hernández I, Valero S, Alegret M, Ruiz A, et al. Design of a comprehensive Alzheimer's disease clinic and research center in Spain to meet critical patient and family needs. Alzheimers Dement J Alzheimers Assoc. 2014;10(3):409-15.

39. Nordberg A, Carter SF, Rinne J, Drzezga A, Brooks DJ, Vandenberghe R, et al. A European multicentre PET study of fibrillar amyloid in Alzheimer's disease. Eur J Nucl Med Mol Imaging. 2013;40(1):104-14.

40. Hixson JE, Vernier DT. Restriction isotyping of human apolipoprotein $E$ by gene amplification and cleavage with Hhal. J Lipid Res. 1990;31(3):545-8.

41. Insel PS, Mattsson N, Mackin RS, Schöll M, Nosheny RL, Tosun D, et al. Accelerating rates of cognitive decline and imaging markers associated with B-amyloid pathology. Neurology. 2016:86(20):1887-96.

42. Schupf N, Tang MX, Fukuyama H, Manly J, Andrews H, Mehta P, et al. Peripheral A subspecies as risk biomarkers of Alzheimer's disease. Proc Natl Acad Sci. 2008;105(37):14052-7.
43. Swaminathan S, Risacher SL, Yoder KK, West JD, Shen L, Kim S, et al. Association of plasma and cortical amyloid beta is modulated by APOE $\varepsilon 4$ status. Alzheimers Dement. 2014;10(1):e9-18.

44. Furst AJ, Rabinovici GD, Rostomian AH, Steed T, Alkalay A, Racine C, et al. Cognition, glucose metabolism and amyloid burden in Alzheimer's disease. Neurobiol Aging. 2012;33(2):215-25.

45. La Joie R, Perrotin A, Barré L, Hommet C, Mézenge F, Ibazizene M, et al. Region-specific hierarchy between atrophy, hypometabolism, and $\beta$-amyloid $(A \beta)$ load in Alzheimer's disease dementia. J Neurosci. 2012;32(46):16265-73.

46. Tauber C, Beaufils E, Hommet C, Ribeiro MJ, Vercouillie J, Vierron E, et al. Brain [18F]FDDNP binding and glucose metabolism in advanced elderly healthy subjects and Alzheimer's disease patients. J Alzheimers Dis. 2013; 36(2):311-20.

47. Hatashita S, Yamasaki H, Suzuki Y, Tanaka K, Wakebe D, Hayakawa H. [18F]Flutemetamol amyloid-beta PET imaging compared with [11C]PIB across the spectrum of Alzheimer's disease. Eur J Nucl Med Mol Imaging. 2014;41(2):290-300

48. Wirth M, Oh H, Mormino EC, Markley C, Landau SM, Jagust WJ. The effect of amyloid $\beta$ on cognitive decline is modulated by neural integrity in cognitively normal elderly. Alzheimers Dement J Alzheimers Assoc. 2013; 9(6):687-698.e1.

49. Mosconi L, Andrews RD, Matthews DC. Comparing brain amyloid deposition, glucose metabolism, and atrophy in mild cognitive impairment with and without a family history of dementia. J Alzheimers Dis. 2013;35(3): 509-24.

50. Lowe VJ, Weigand SD, Senjem ML, Vemuri P, Jordan L, Kantarci K, et al. Association of hypometabolism and amyloid levels in aging, normal subjects. Neurology. 2014;82(22):1959-67.

51. Kadir A, Almkvist $\mathrm{O}$, Forsberg A, Wall A, Engler $H$, Långström B, et al. Dynamic changes in PET amyloid and FDG imaging at different stages of Alzheimer's disease. Neurobiol Aging. 2012;33(1):198.e1-14.

52. Frings L, Spehl TS, Weber WA, Hüll M, Meyer PT. Amyloid- $\beta$ load predicts medial temporal lobe dysfunction in Alzheimer dementia. J Nucl Med Off Publ Soc Nucl Med. 2013;54(11):1909-14.

53. Edison $P$, Archer HA, Hinz R, Hammers A, Pavese N, Tai YF, et al. Amyloid, hypometabolism, and cognition in Alzheimer disease: an [11C]PIB and [18F]FDG PET study. Neurology. 2007:68(7):501-8.

54. Zhou Y, Yu F, Duong TQ. Alzheimer's Disease Neuroimaging Initiative. White matter lesion load is associated with resting state functional MRI activity and amyloid PET but not FDG in mild cognitive impairment and early Alzheimer's disease patients. J Magn Reson Imaging. 2015;41(1):102-9.

55. Drzezga A, Becker JA, Van Dijk KRA, Sreenivasan A, Talukdar T, Sullivan C, et al. Neuronal dysfunction and disconnection of cortical hubs in nondemented subjects with elevated amyloid burden. Brain J Neurol. 2011; 134(Pt 6):1635-46.

\section{Publisher's Note}

Springer Nature remains neutral with regard to jurisdictional claims in published maps and institutional affiliations.

\section{Ready to submit your research? Choose BMC and benefit from:}

- fast, convenient online submission

- thorough peer review by experienced researchers in your field

- rapid publication on acceptance

- support for research data, including large and complex data types

- gold Open Access which fosters wider collaboration and increased citations

- maximum visibility for your research: over $100 \mathrm{M}$ website views per year

At BMC, research is always in progress.

Learn more biomedcentral.com/submission 